\title{
Article
}

\section{Interaction of Metallic Nanoparticles with \\ a Biologically Active Molecule, Dopamine}

Chunhui Liu, Haiying He, and Ravindra PandeySaber HussainShashi P. Karna

J. Phys. Chem. B, 2008, 112 (47), 15256-15259 • DOI: 10.1021/jp808009t • Publication Date (Web): 05 November 2008

Downloaded from http://pubs.acs.org on December 23, 2008

\section{More About This Article}

Additional resources and features associated with this article are available within the HTML version:

- $\quad$ Supporting Information

- $\quad$ Access to high resolution figures

- $\quad$ Links to articles and content related to this article

- $\quad$ Copyright permission to reproduce figures and/or text from this article

\section{View the Full Text HTML}




\title{
Interaction of Metallic Nanoparticles with a Biologically Active Molecule, Dopamine
}

\author{
Chunhui Liu, Haiying He, and Ravindra Pandey* \\ Department of Physics and Multi-Scale Technologies Institute, Michigan Technological University, \\ Houghton, Michigan 49931, USA
}

\author{
Saber Hussain \\ US Air Force Research Laboratory, Dayton, Ohio
}

\author{
Shashi P. Karna \\ US Army Research Laboratory, Aberdeen Proving Ground, Maryland 21005
}

Received: September 9, 2008

\begin{abstract}
We present the results of first-principles molecular orbital calculations describing the interaction of metallic nanoparticles, represented by $\mathrm{Mn}_{13}, \mathrm{Ag}_{13}$, and $\mathrm{Al}_{13}$ atomic clusters, with a biologically active molecule, dopamine. The interaction strength, determined in terms of the nanoparticle-molecule complex binding energy, is found to be higher for $\mathrm{Mn}$ than either $\mathrm{Ag}$ or $\mathrm{Al}$ and can be explained in terms of the degree of the hybridization of the (metal) atomic orbitals with the molecular orbitals in the complex. Furthermore, smaller interaction strength of these metallic nanoparticles with water compared to that with dopamine predicts the preference of forming a complex of dopamine with the metallic nanoparticles in the aqueous solution. The calculated results may therefore suggest that the presence of these metallic nanoparticles could induce different levels of dopamine depletion in solution.
\end{abstract}

\section{Introduction}

Engineered nanomaterials with at least one dimension within the size range of $1-100$ nanometers are increasingly finding commercial applications as fillers, UV blockers, light reflectors, catalysts, semiconductors, and light emitters. ${ }^{1,2}$ The unique chemical and physical properties, such as chemical reactivity, thermal and electrical conductivity, and optical sensitivity of engineered nanomaterials combined with the fact that such properties can be further tailored by engineering the size, shape, and chemical composition make these materials highly desirable for a wide variety of commercial as well as noncommercial technologies and consumer products. However, with increased activities in their synthesis, growth, and integration in technology and consumer products, there has also been increased concern about their biological and environmental effects. ${ }^{3}$ Therefore, as the production and applications of engineered nanomaterials continue to increase, it is deemed critically important and timely to develop an understanding of their health and environmental effects and to develop a baseline safety guidance of their possible toxicity.

Recently, several experimental studies were devoted to understand the toxicity of nanoparticles, such as diamond, ${ }^{4} \mathrm{C}_{60},{ }^{5}$ and silica nanoparticles in human lung cancer cells, ${ }^{6}$ iron oxide nanoparticles internalization in growing neurons, ${ }^{7}$ and manganese oxide and silver nanoparticles within PC- 12 cells. ${ }^{8}$ A caseby-case difference was suggested from these experiments. For example, nanodiamonds were found to have no toxic effects on a variety of cell types, whereas cytotoxicity was observed for $\mathrm{C}_{60}$, silica, and iron oxide nanoparticles, where exposure to increasing concentration of these nanoparticles results in a dose-

* Corresponding author: e-mail: pandey@mtu.edu; phone: +1 (906) 4872086; fax: +1 (906) 487-2933. dependent diminishing viability and capacity of living cells. On the other hand, the toxic reactivity in terms of the level of dopamine in PC-12 cells was found to be higher for Mn than that for Ag nanoparticles. ${ }^{8}$

The importance of the extraordinary characteristics of the nanoparticles themselves in toxicity is often omitted: small size, high surface area, high reactivity, and altered electronic structure. ${ }^{9}$ The aim of this paper is to understand the reaction of metallic nanoparticles with a biological active molecule: dopamine. The propensity of nanoparticles forming a complex with dopamine in the aqueous solution may lead to the reduction of the concentration of free dopamine molecules in the solution. As a result, it may also be one of the causes of the dopamine depletion in cells that are cultured in such a solution. We have performed first-principles quantum mechanical calculations to obtain the energy surface describing the interaction of metallic nanoparticles with the dopamine molecule. Specifically, we have performed electronic structure calculations on a dopamine molecule interacting with atomic clusters of manganese and silver, represented by $\mathrm{Mn}_{13}$ and $\mathrm{Ag}_{13}$, respectively. Additionally, we have also considered atomic clusters of aluminum (e.g., $\mathrm{Al}_{13}$ ) due to the commercial use of aluminum nanoparticles in solid rocket fuel ${ }^{10}$ and conductive coatings. ${ }^{11}$ The calculated results provide very interesting and useful information on relative strength as well as the site specificity of metallic nanoparticledopamine binding. It is expected that the present study not only provides a better understanding of the underlying mechanism of dopamine depletion by metal nanoparticles, but would also provide guidance to establish a comprehensive kinetic model describing the nanoparticle-molecule interaction.

\section{Computational Method}

All calculations reported here were performed within the framework of the density functional theory (DFT). We used 


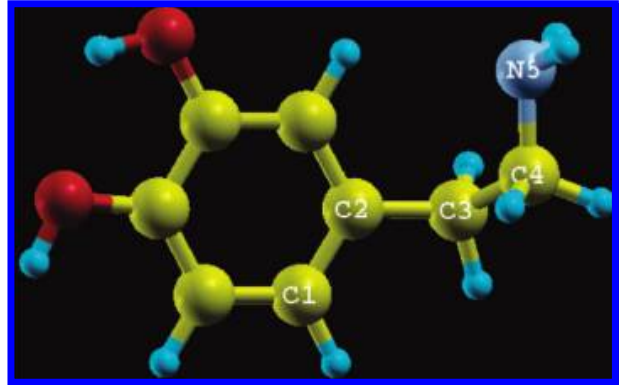

Figure 1. A ball-and-stick model for the dopamine molecule (O: red, C: yellow, N: navy blue, H: blue).

the Vienna ab initio simulation package (VASP) code employing the projector augmented-wave (PAW) potentials. ${ }^{12-14}$ The exchange and correlation functional forms proposed by Perdew and Zunger ${ }^{15,16}$ were used in the generalized gradient approximation (GGA) to density functional theory. We note here that the use of GGA approximation was found to be essential for magnetic metallic clusters, such as Mn clusters, to obtain an accurate and reliable energy surface. ${ }^{16}$

The computational parameters were taken from our previous studies on $\mathrm{Ni}_{n} \mathrm{~B}$ clusters. ${ }^{17}$ For example, atoms in a given cluster were positioned in a cubic supercell with an edge of $20 \AA$. The cutoff energy for the plane wave basis was set to $400 \mathrm{eV}$. The electronic structure calculations were considered to be converged when the force on each ion was less than $0.03 \mathrm{eV} / \AA$. Additionally, the total energy convergence criterion of $10^{-5} \mathrm{eV}$ was used.

\section{Results and Discussion}

3.1. Dopamine. The optimized equilibrium structure for the dopamine molecule is shown in Figure 1. The two hydroxyl $(-\mathrm{OH})$ groups are nearly coplanar with the phenyl ring and form an $\mathrm{O}-\mathrm{H} \cdots \mathrm{O}-\mathrm{H}$ intramolecular hydrogen bond in their most stable arrangement. This is consistent with an earlier study. ${ }^{18}$ The lowest-energy structure found in this study has the characteristic dihedral angles (as defined in the literatures $18-20)$ of $\varphi_{1}(\mathrm{~N} 5-\mathrm{C} 4-\mathrm{C} 3-\mathrm{C} 2)=74.09^{\circ}, \varphi_{2}(\mathrm{C} 4-\mathrm{C} 3-$ $\mathrm{C} 2-\mathrm{C} 1)=116.92^{\circ}$ following the gauche conformer configuration $\left(60^{\circ}, 90^{\circ}\right)$ for the $\left(\varphi_{1}, \varphi_{2}\right)$ pair. ${ }^{19}$ The calculated geometrical parameters are in reasonable agreement with the results of an earlier study ${ }^{20}$ giving the gauche conformer configuration for $\mathrm{N}$-protonated dopamine with the values of $\left(54.60^{\circ}, 85.03^{\circ}\right)$. However, we also find that only a small difference in the orientation of the $-\mathrm{CH} 2-\mathrm{CH}_{2}-\mathrm{NH}_{2}$ tail can yield different structural isomers for the ground state, thereby confirming the flexibility of the rotation of the $\mathrm{C}-\mathrm{C}$ and $\mathrm{C}-\mathrm{N}$ $\sigma$-bonds in the molecule. ${ }^{19,20}$

3.2. $\mathbf{M n}_{13}, \mathbf{A g}_{13}$, and $\mathbf{A l}_{13}$ Nanoparticles. The ground-state of a 13-atom metallic nanoparticle is, generally, predicted to be an icosahedra followed by either hexagonal close packed or cuboctahedral configurations. This is the case with the nanopartciles considered, namely $\mathrm{Mn}_{13}, \mathrm{Ag}_{13}$, and $\mathrm{Al}_{13}$, as also reported in the previous studies. ${ }^{21-28}$ For $\mathrm{Mn}_{13}$, the ground-state prefers antiferromagnetic ordering as indicated in Figure 2. In contrast to $\mathrm{Mn}_{13}, \mathrm{Al}_{13}$ and $\mathrm{Ag}_{13}$ have the doublet spin state in the ground-state due to the odd-electron system. ${ }^{29}$ The (average) bond lengths of these metallic atomic clusters are listed in Table 1 , showing a very good agreement with previously reported values. $^{21-29}$

3.3. Interaction of Metallic Nanoparticles with Dopamine. We begin with the icosahedral configuration of metallic atomic clusters and consider the cluster to approach the molecule toward the oxygen atoms (i.e., the $\mathrm{O}$-site), nitrogen (i.e., the $\mathrm{N}$-site),

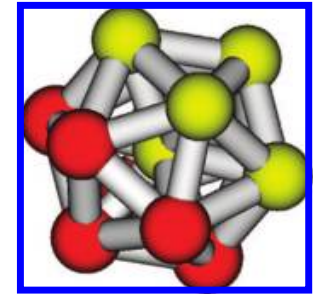

Figure 2. The ground-state configuration of $\mathrm{Mn}_{13}$. The atoms with spin up state are green, and the atoms with spin down are red.

TABLE 1: Bond Lengths of $\mathbf{M n}_{13}, \mathrm{Ag}_{13}$, and $\mathrm{Al}_{13}$

\begin{tabular}{ccc}
\hline & \multicolumn{2}{c}{ (average) bond length $(\AA)$} \\
\cline { 2 - 3 } cluster & this work & other work ${ }^{21-29}$ \\
\hline $\mathrm{Mn}_{13}$ & 2.52 & 2.54 \\
$\mathrm{Ag}_{13}$ & 2.86 & 2.87 \\
$\mathrm{Al}_{13}$ & 2.52 & 2.59
\end{tabular}

and the hexagon ring (i.e., the top-site) of the dopamine molecule (Figure 1). The paths approaching to the $\mathrm{O}$ - and $\mathrm{N}$-sites are constrained on the plane of the molecule whereas the path going to the top-site is constrained perpendicular to the plane of the molecule. Note that the molecular coordinates are relaxed as the metallic cluster approaches the molecule. The calculated energy surface for $\mathrm{Mn}_{13}, \mathrm{Ag}_{13}$, and $\mathrm{Al}_{13}$ are shown in Figures 3,4 , and 5 , respectively. Here, the distance is taken to be the distance between the nearest cluster atom facing the molecule and the approaching molecular site. The reference point of energy is taken to be the energy of the system when the cluster and the molecule are far apart from each other. The binding

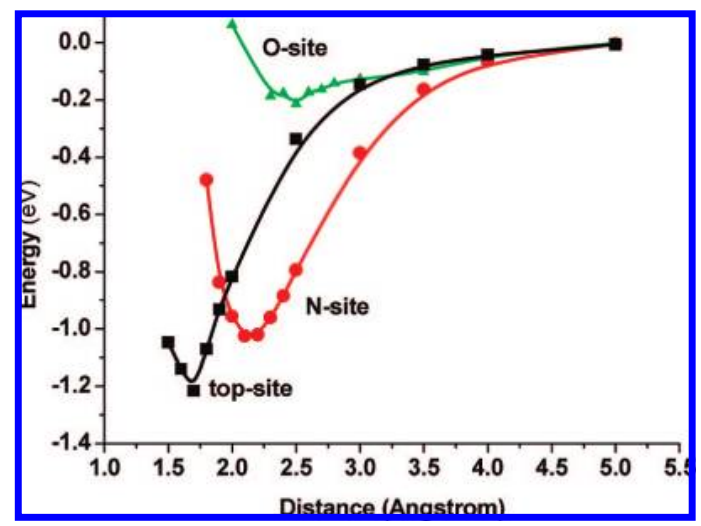

Figure 3. The energy surface describing interaction of $\mathrm{Mn}_{13}$ with a dopamine molecule.

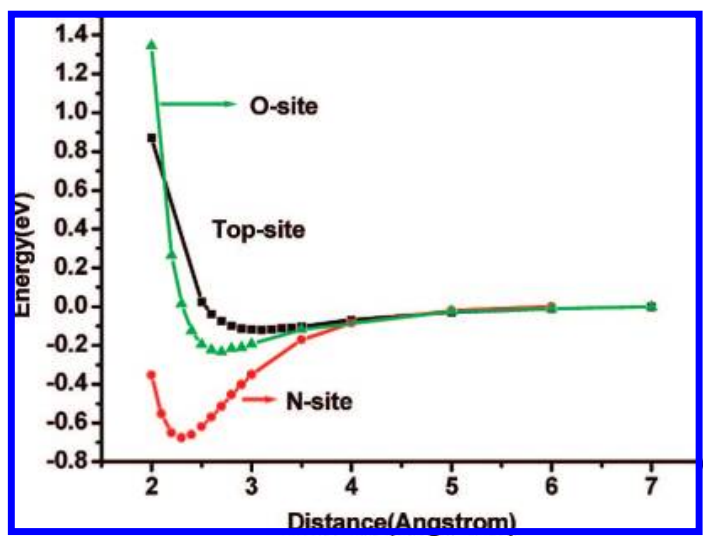

Figure 4. The energy surface describing interaction of $\mathrm{Ag}_{13}$ with a dopamine molecule. 


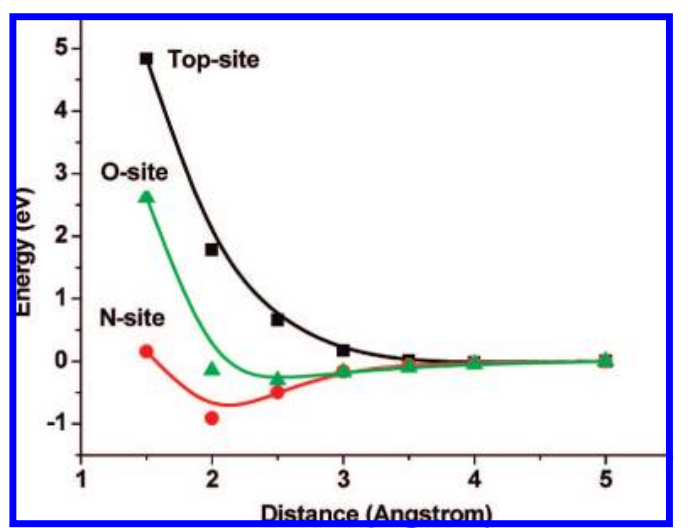

Figure 5. The energy surface describing interaction of $\mathrm{Al}_{13}$ with a dopamine molecule.

TABLE 2: Binding Energy $\left(E_{B}\right)$ and Distance $\left(R_{B}\right)$ of the Nanoparticle-Molecule Complex

\begin{tabular}{lllll}
\hline & & \multicolumn{3}{c}{ molecular sites } \\
\cline { 3 - 5 } \multicolumn{2}{c}{ metallic nanopartcile } & N-site & O-site & top-site \\
\hline $\mathrm{Mn}_{13}$ & $E_{\mathrm{B}}, \mathrm{eV}$ & 1.02 & 0.42 & 1.22 \\
& $R_{\mathrm{B}}, \AA$ & 2.1 & 2.5 & 1.7 \\
$\mathrm{Ag}_{13}$ & $E_{\mathrm{B}}, \mathrm{eV}$ & 0.67 & 0.27 & 0.12 \\
& $R_{\mathrm{B}}, \AA$ & 2.3 & 2.7 & 3.1 \\
$\mathrm{Al}_{13}$ & $E_{\mathrm{B}}, \mathrm{eV}$ & 0.91 & 0.30 & not bound \\
& $R_{\mathrm{B}}, \AA$ & 2.0 & 2.5 &
\end{tabular}

energy of the nanoparticle-molecule system is then calculated as the difference of the energy associated with the equilibrium configuration and the energy associated with the reference point. For example, the binding energy for the $\left(\mathrm{Mn}_{13}+\right.$ molecule $)$ complex is the difference in total energy of the configurations at the separations of 5.0 and $1.7 \AA$, respectively.

The calculated values of the binding energy $\left(E_{\mathrm{B}}\right)$ and the distance $\left(R_{\mathrm{B}}\right)$ associated with the ground-state configuration of the complex consisting of the nanoparticle and molecule are given in Table 2.

The energy surface does not show the presence of the energy barrier for all the cases considered (Figures 3-5), thus indicating the likelihood of exothermic reaction between metallic atomic clusters and the dopamine molecule. For $\mathrm{Mn}_{13}$, the calculated results show the preference for the top-site with the binding energy of $1.22 \mathrm{eV}$. It is closely followed by the preference for the N-site and O-site with the binding energies of 1.02 and 0.42 $\mathrm{eV}$, respectively. However, this is not the case of either $\mathrm{Ag}_{13}$ or $\mathrm{Al}_{13}$, where the preferred binding site is predicted to be the $\mathrm{N}$-site followed by the O-site and the top-site. The predicted interaction strength at the $\mathrm{N}$-site of the molecule is much higher than that at the $\mathrm{O}$-site, although the metallic atoms tend to bind more strongly with the oxygen atom relatively to the biding with a nitrogen atom. This is due to the fact that the so-called $\mathrm{O}$-site is the bridge site to two $\mathrm{O}$ atoms each bonded to two other atoms in the molecule. Furthermore, the calculated values of the binding energy for $\mathrm{Ag}_{13}$ and $\mathrm{Al}_{13}$ are significantly lower than those associated with $\mathrm{Mn}_{13}$. The significantly higher interaction strength of $\mathrm{Mn}_{13}$, therefore, suggests that the manganese nanoparticles, and not the silver or aluminum nanoparticles, are likely to be associated with the higher level of free dopamine depletion. Calculations are in progress to develop a comprehensive kinetic model representing reactivity of metallic nanoparticle with the dopamine molecule.

Finally, we show the charge density plots (Figure 6) of the nanoparticle-molecule complex to explain the difference in interaction strength together with the site selectivity shown by
$\mathrm{Mn}_{13}, \mathrm{Ag}_{13}$, and $\mathrm{Al}_{13}$. In the equilibrium configuration of the nanoparticle-molecule complex, the top-site among the three molecular sites facilitates the maximum overlap of electron clouds of the atomic cluster and molecule, since all of the atoms constituting the molecule are likely to participate in the bonding. Because the electronic configuration of the $\mathrm{Mn}$ atom is $3 \mathrm{~d}^{5} 4 \mathrm{~s}^{2}$, the molecular levels of $\mathrm{Mn}_{13}$ arising from the Mn 4s orbitals can be considered to be filled. A small interaction will split the $\mathrm{Mn} 3 \mathrm{~d}$ orbitals in a crystal field-like manner: in the $\mathrm{Mn}_{13}$ cluster, the central $3 \mathrm{~d}$ orbitals form a single degenerate representation of $h_{g}$ character within the $I_{h}$ group, while the surface $3 \mathrm{~d}$ orbitals will split, within their $C_{5 v}$ symmetry, into $e_{1}\left(\mathrm{~d}_{x z}, \mathrm{~d}_{y z}\right), e_{2}\left(\mathrm{~d}_{x^{2}}-y^{2}\right.$, $\left.\mathrm{d}_{x y}\right)$, and $a_{l}\left(\mathrm{~d}_{z^{2}}\right)$. Because the central-to-surface atomic distance is shorter than the surface-to-surface atom distance, the ordering of these almost degenerate orbitals will be $e_{1}<e_{2}<a_{1}<h_{g}$. In $\mathrm{Mn}_{13}$, filling these orbitals with the $65 \mathrm{~d}$ electrons will yield the electronic configuration of $e_{1}{ }^{48} e_{2}{ }^{17} a_{1}{ }^{0} h_{g}{ }^{0}$, with nonfully occupied d orbitals. This is further confirmed by the calculated orbital-projected density of states of $\mathrm{Mn}_{13}$, not shown here.

When the Mn atoms interact with the dopamine molecule, the resulting electronic configuration of the nanoparticle-molecule complex will be different. While interacting from the top of the phenyl ring, the $\pi$ orbitals of phenyl will undergo a strong hybridization with their environment, thus lowering their energy. These mixed orbitals will be filled before the remaining Mn 3d orbitals, such as $\mathrm{Mn} h_{g}$. Overall, the orbital pattern consists of (i) symmetry-split, almost degenerate, $3 \mathrm{~d}$ orbitals versus (ii) mostly hybridized $\mathrm{Mn}-3 \mathrm{~d}$ and phenyl- $\pi$ orbitals that are lower in energy. In the nanoparticle-molecule complex, the charge density plots at different contour values show a strong overlap of the electron clouds associated with the metallic atoms and the molecule. The higher interactive strength of $\mathrm{Mn}_{13}$ with dopamine can then be attributed to the great degree of hybridization that forces the pairing of the electrons in the mixed orbitals of Mn and dopamine.

In contrast, the electronic configuration of the $\mathrm{Ag}$ atom is $4 \mathrm{~d}^{10} 5 \mathrm{~s}^{1}$, and $\mathrm{Ag}_{13}$ is characterized by $s$-type frontier orbitals, with one unpaired electron in the odd-number atomic cluster. The Ag-s orbitals undergo a smaller hybridization with the phenyl- $\pi$ orbitals, yielding a weaker interactive strength with the molecule. Likewise, the $\mathrm{Al}$ atom has an electronic configuration of $3 \mathrm{~s}^{2} 3 \mathrm{p}^{1}$, and the icosahedral-like $\mathrm{Al}_{13}$ cluster falls into the case where the 12 surface atoms form a close-shell electronic configuration similar to that of $\mathrm{Kr}$ with 36 electrons in total, while the central atom has an unpaired electron with p-type character. ${ }^{23}$ When the atomic cluster approaches the dopamine molecule from the top-site, an overall Coulomb repulsion is noted due to the closed shell of $\mathrm{Al}_{13}$ cluster. Therefore, no bonding is detectable with the molecule, as shown by the repulsive potential energy surface in Figure 5.

Considering the fact that the experimental studies were performed on the biological cells in the water solution, we have further studied the interaction of metallic nanoparticles with water. The calculated energy surfaces are shown in Figure 7, where the distance is taken from the outmost atom facing the molecule to the $\mathrm{O}$ atom of water and the energy at the $7 \AA$ is aligned to zero as a reference point. Accordingly, the binding energy of the $\mathrm{Mn}_{13}$ +water and $\mathrm{Ag}_{13}+$ water complexes comes out to be 0.47 and $0.24 \mathrm{eV}$, respectively with the equilibrium distance of 2.1 and $2.4 \AA$, respectively.

Because a simulation of the interaction of the metallic nanoparticles with the dopamine molecule in the aqueous environment is beyond the scope of this study, we estimate the relative strengths of the two competing reaction processes (i.e., 


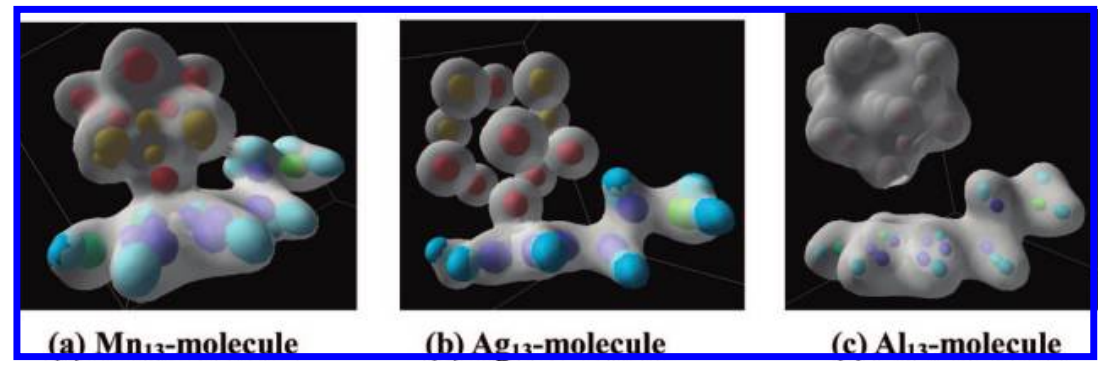

Figure 6. The charge density of the cluster-molecule complex showing the top-site interaction: (a) $\mathrm{Mn}_{13}$, (b) $\mathrm{Ag}_{13}$, (c) $\mathrm{Al}_{13}$. The contour density for (a) and (b) is $0.32 \mathrm{e} / \AA^{3}$, and that for (c) is $0.16 \mathrm{e} / \AA^{3}$.

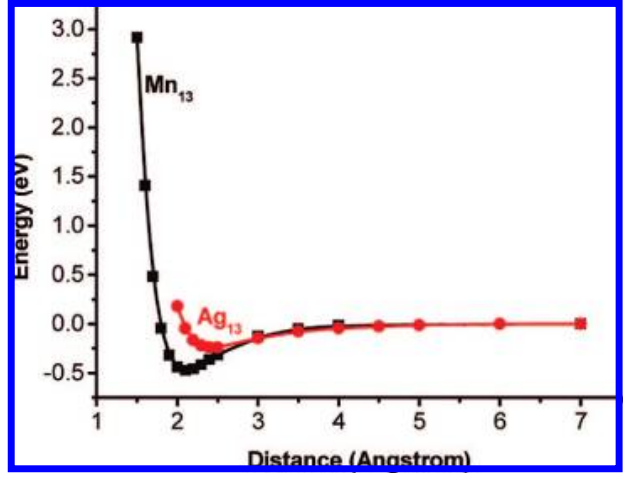

Figure 7. The energy surface describing interaction of $\mathrm{Mn}_{13}$ and $\mathrm{Ag}_{13}$ with water.

interaction of metallic nanoparticles with a dopamine molecule or with a water molecule) by comparing their binding energies of the respective complexes. Interestingly, a significantly higher binding energy for the $\mathrm{Mn}_{13}+$ dopamine complex relative to $\mathrm{Mn}_{13}+$ water complex points toward the fact that metallic Mn nanoparticles most probably form a complex with the dopamine molecule instead of water, thus leading to the concentrationdependent depletion of dopamine in the aqueous solution. Likewise, the relatively stronger interaction of Ag with dopamine than with water suggests the same effect of depletion of dopamine in the aqueous solution. However, this depletion effect in the presence of Ag nanoparticles is likely to be smaller than that in the presence of $\mathrm{Mn}$ nanoparticles, due to relatively smaller interaction strength of $\mathrm{Ag}$ nanoparticles.

\section{Summary}

We have investigated the interaction of metallic nanoparticles, represented by $\mathrm{Mn}, \mathrm{Ag}$, and $\mathrm{Al}$ atomic clusters, with a biologically active molecule, dopamine. The interaction strength in terms of the metallic nanoparticles-dopamine binding energy is predicted to be higher for $\mathrm{Mn}$ relative to either $\mathrm{Ag}$ or $\mathrm{Al}$ nanopaticles. We also find that $\mathrm{Mn}_{13}$ prefers to bind with the top-site, whereas $\mathrm{Ag}_{13}$ and $\mathrm{Al}_{13}$ prefer to bind with the $\mathrm{N}$-site of the molecule. The site selectivity and the interaction strength of metallic nanoparticles can be explained in terms of the degree of the hybridization of the (metal) atomic orbitals with the molecular orbitals in the cluster-molecule complex. The smaller interaction strength of these metallic nanoparticles with water compared to that with dopamine further confirms the preferred adsorption of dopamine onto their surface in the aqueous solution. The higher interaction strength predicted for $\mathrm{Mn}_{13}$ relative to the $\mathrm{Ag}_{13}$ may suggest a higher level of dopamine depletion in solution due to this binding mechanism alone in the presence of $\mathrm{Mn}$ and $\mathrm{Ag}$ nanoparticles. We have no means, however, to declare that this is the only toxic pathology and mechanism for these nanoparticles existing in a cell culture media. The results also form a basis for calculations which are now in progress to make a comprehensive kinetic model describing the reactivity of the nanoparticle-molecule complex.

Acknowledgment. Helpful discussions with R. Scheicher, S. Gowtham, and K. C. Lau are acknowledged. The work at Michigan Technological University was supported by DARPA (contract No. ARL-DAAD17-03-C-0115).

\section{References and Notes}

(1) Xia, Y.; Yang, P.; Sun, Y.; Wu, Y.; Mayers, B.; Gates, B.; Yin, Y.; Kim, F.; Yan, H. Adv. Mater. 2003, 15, 353.

(2) Xu, T. T.; Fisher, F. T.; Cate Brinson, L.; Ruoff, R. S. Nano Lett. 2003, 3, 1135 .

(3) Bai, R.; Wang, W.; Jin, X.-L. J. Environ. Health 2007, 24, 59.

(4) Schrand, A. M.; Huang, H.; Carlson, C.; Schlager, J. J.; Omacr Sawa, E.; Hussain, S. M.; Dai, L. J. Phvs. Chem. B 2007, 111 (1), 2.

(5) Oberdörster, E. Environ. Health Perspect. 2004, 112 (10), 1058.

(6) W. Lin, Y. W.; Huang, X. D.; Zhou, Y. Ma. Toxicol. Appl. Pharmacol. 2007, 15, 220.

(7) Pisanic, T. R.; Blackwell, J. D.; Shubayev, V. I.; Fiñones, R. R.; Jin, S. Biomaterials 2007, 28 (16), 2572.

(8) Saber, M.; Hussain, K.; Javorina, A. M.; Schrand, A. M.; Duhart, H.; Ali, F. S.; Schlager, J J. Toxicol. Sci. 2006, 92 (2), 456.

(9) Nel, A.; Xia, T.; Mädler, L.; Li, N. Science 2006, 311, 622.

(10) Park, K.; Lee, D.; Rai, A.; Mukherjee, D.; Zachariah, M. R. J. Phvs. Chem. B 2005, 109, 7290 .

(11) Kun., Luo; Nanlin, Shi; Hongtao, Cong; Chao, Sun J. Solid State Electrochem. 2006, 10, 1003.

(12) Kresse, G.; Furthmüller, J. Phvs. Rev. B 1996, 54, 11169.

(13) Blöchl, P. E. Phvs. Rev. B 1994, 50, 17953.

(14) Kresse, G.; Joubert, D. Phys. Rev. B 1999, 59, 1758.

(15) Perdew, J. P.; Zunger, A. Phvs. Rev. B 1981, 23, 5048.

(16) Perdew, J. P.; Chevary, J. A.; Vosko, S. H.; Jackson, K. A.; Pederson, M. R.; Singh, D. J.; Fiolhais, C. Phys. Rev. B 1992, 46, 6671.

(17) Deshpande, M. D.; Kanhere, D. G.; Pandey, R. Phvs. Rev. A 2005, 71,63202 .

(18) Alagona, G.; Ghio, C. Chem. Phvs. 1996, 204, 239.

(19) Urban, J. J.; Cramer, C. J.; Famini, G. R. J. Am. Chem. Soc. 1992, 114,8226 .

(20) Nagy, P. I.; Alagona, G.; Ghio, C. J. Am. Chem. Soc. 1999, 121, 4804 .

(21) Hafner, J.; Hobbs, D. Phvs. Rev. B 2003, 68, 014408.

(22) Knickelbein, M. B. Phvs. Rev. B 2004, 70, 014424.

(23) Kabir, M.; Mookerjee, A. Phvs. Rev. B 2006, 73, 224439.

(24) Briere, T. M.; F Sluiter, M. H.; Kumar, V.; Kawazoe, Y. Bull. Mater. Sci. 2003, 26, 115.

(25) Michaelian, K.; Rendón, N.; Garzón, I. L. Phys. Rev. B 1999, 60, 2000.

(26) Pereiro, M.; Baldomir, D. Phvs. Rev. A 2007, 75, 33202.

(27) Arslan, H.; Güven, M. H. New J. Phys. 2005, 7, 60.

(28) Majumder, C.; Kulshreshtha, S. K. Chem. Phvs. Lett. 2000, 323, 393.

(29) Liu, R. S.; Liu, F. X.; Zhou, Q. Y.; Wang, X.; Zheng, C. X. Solid State Phenomena 2007, 121, 1139-123.

JP808009T 